\title{
Effects of ipsilateral rostral pontine reticular lesions on retention of classically conditioned nictitating membrane and eyelid responses
}

\author{
DAVID G. LAVOND, DAVID A. McCORMICK, GREGORY A. CLARK, \\ DAVID T. HOLMES, and RICHARD F. THOMPSON \\ Stanford University, Stanford, California 94305
}

\begin{abstract}
Neural unit recordings from the pontine reticular formation in well-trained rabbits show a tone-evoked onset response (CS), a suggestion of an airpuff onset response (UCS), and an increase in unit activity that is similar to the topography of the learned response. When this same area is destroyed by unilateral electrolytic lesions (in another group of animals), retention of the learned response is abolished but the reflex response is essentially unchanged. Since a recent study (McCormick, Lavond, Clark, Kettner, Rising, \& Thompson, 1981) has shown that unilateral cerebellar lesions also produce a complete and selective abolition of the CR, and the CR cannot be reinstated with retraining, these findings are discussed in terms of a common circuitry that encodes the engram for short-delay classical conditioning of striated muscle responses.
\end{abstract}

The nature of the engram-the neuronal substrate of learning and memory-has proved to be one of the most baffling problems in science. Our approach has been to adopt a particularly clear and well-controlled form of associative learning-classical conditioning of the rabbit nictitating membrane (NM) response (Gormezano, Schneiderman, Deaux, \& Fuentes, 1962) - as a "simple"model system in which to analyze the neuronal mechanisms of learning.

A number of brain structures appear to play important roles in even this presumably very simple form of learning. Thus, spreading depression of the contralateral face area of the motor cortex of the rabbit reversibly abolishes the standard short-delay CR-a powerful effect on memory retrieval (Megirian \& Bureš, 1970; Papsdorf, Longman, \& Gormezano, 1965). Neuronal activity in the hippocampus becomes massively engaged early in training and invariably and accurately predicts the subsequent development of behavioral learning over a wide range of conditions (Thompson, Berger, Berry, Hoehler, Kettner, \& Weisz, 1980). Furthermore, bilaterally induced seizures or disruptive electrical stimulation of the hippocampus prevent or severely impair initial learning of the short-delay CR (Salafia, Romano, Tynan, \& Host, 1977; Thompson et al., 1980). Finally, if greater demands are made on the learning-memory system, as in trace conditioning, latent inhibition, or discrimination reversal, animals with prior ablation of the hippocampus are unable to learn (Solomon, 1980; Weisz, Solomon, \& Thompson, 1980). However, ani-

Supported in part by Research Grant BNS-8106648 from the National Science Foundation. We thank Jann Lincoln for assistance in histology. mals with prior ablations of the hippocampus (Solomon \& Moore, 1975) or neocortex (Oakley \& Russell, 1972) are able to learn the standard short-delay CR relatively normally. In fact, rabbits with all brain tissue above the level of the thalamus removed are able to learn the short-delay CR (NM response; see Enser, Note 1), as are decerebrate cats (eyeblink; see Norman, Buchwald, \& Villablanca, 1977).

Although many alternative inferences are possible from these observations, we have adopted the following as a working hypothesis: There is an essential circuitry in the midbrain-brainstem that forms the neuronal substrate of the short-delay CR. Higher brain systems normally play very important modulatory roles, which become essential under more demanding conditions of learning and memory.

If there is an essential learning circuitry in the midbrain-brainstem, it should be possible to eliminate the CR selectively by a relatively restricted lesion. The first question is to identify the putative learning circuitry. We have approached this question by undertaking a detailed electrophysiological mapping of neural unit activity throughout the midbrainbrainstem in trained animals (McCormick, Lavond, $\&$ Thompson, Note 2 ). To date, we have tentatively identified at least two candidates for the learning circuitry in terms of learning-related increases in unit activity: (1) a system in the pontine reticular tegmentum, and (2) the lateral portion of the cerebellum. In a recent paper, we reported that ipsilateral lesions of the lateral neocerebellum completely and permanently abolished the short-delay conditioned response but had no effect on the reflex response (McCormick, Lavond, Clark, Kettner, Rising, \& Thompson, 1981). In this paper, we report preliminary results of elec- 
trophysiological recording and focus on effects of lesions in the reticular region. In brief, relatively limited ipsilateral lesions of this region produce a selective abolition of the short-delay CR.

In this paradigm, the measured response is an extension of the NM. However, the conditioned response is in fact a coordinated response involving NM extension, which is largely a passive result of eyeball retraction (Cegavske, Thompson, Patterson, \& Gormezano, 1976; Motais, 1885, cited in Bach-yRita, 1971), external eyelid closure, and contraction of the periorbital facial musculature, the major components being the NM and eyelid responses (Lavond, Judson, \& Thompson, Note 3; McCormick \& Thompson, Note 4). The effects reported here occur for both the NM and eyelid components of the conditioned response.

\section{METHOD}

Data reported here for the lesion experiment included 18 adult male New Zealand white rabbits. The rabbits were housed, fed, and watered under standard laboratory conditions. Each rabbit was first operated upon under halothane anesthesia. The purpose of this operation was to install a chronic micromanipulator base upon the skull above the position at which a movable electrode could later be inserted. Thus, the skull immediately underlying this position was removed and replaced with bone wax. The base was fixed in place with dental acrylic and stainless steel screws. In addition, a headstage was also implanted that would later be used to hold the potentiometer, which measured nictitating membrane (NM) excursion across the eyeball, and also to hold the tube for presentation of the unconditioned stimulus (UCS, i.e., corneal airpuff). Finally, a small loop of nylon suture was placed in the nictitating membrane for later attachment to the potentiometer.

Following 1 week of recovery from the surgery, the rabbits began the training procedure: 1 day of adaptation followed by the number of days of paired training of short-delay conditioning that was necessary for each rabbit to reach criterion and overtraining. The paired training consisted of 13 blocks of one CS-alone test trial (a $1-\mathrm{kHz}$ tone at $85 \mathrm{~dB}$ for $350 \mathrm{msec}$ ) and eight paired trials (with an airpuff of $210 \mathrm{~g} / \mathrm{cm}^{2}$ delivered to the cornea during the last $100 \mathrm{msec}$ of the tone) for a total of 117 trials per day. The intertrial interval ranged from 20 to $40 \mathrm{sec}$, with an average of $30 \mathrm{sec}$ between trials. The rabbits' eyelids were kept open with an eyeclip so that the NM response could be measured without interference (for evidence of simultaneous conditioning of NM and eyelid responses, see Lavond et al., Note 3; McCormick \& Thompson, Note 4). The eyelid component of the response was also observed in some animals in the present experiments. After reaching a criterion of eight conditioned NM responses of more than half a millimeter in amplitude in nine consecutive trials, each rabbit was then overtrained with an additional day of conditioning.

For the recording studies, the chronic micromanipulator permits electrodes to be inserted and placed at virtually any location within approximately $289 \mathrm{~mm}^{3}$ of brain tissue (e.g., a surface area of $12.5 \mathrm{~mm}^{2}$ and depth to $23 \mathrm{~mm}$ ). The recording microelectrode is placed at a given locus where unit activity can be recorded in an already-trained animal, and the animal is given a block of paired trials and one tone-alone trial. The electrode is then advanced, the procedure repeated, and so on. In mapping, we typically record multiple-unit activity, but isolated single-unit recording can also be done using higher impedance microelectrodes. Unit discharges are converted to standard pulses and stored and analyzed in a computer, as described previously (Berger \& Thompson, 1978;
Cegavske, Patterson, \& Thompson, 1979). To date, a total of six animals have been run for mapping of the pontine reticular region.

In the lesion study, reported in detail here, rabbits were anesthetized with halothane immediately following the day of overtraining. Next, a micromanipulator was inserted onto the base that had been implanted during the surgery. A lesion electrode (a size 0 insulated stainless steel insect pin with a .5-mm exposed tip) was then lowered into the approximate location of the reticular formation defined by electrophysiological recordings in the trained animal. The location of this region was approximately AP $10 \mathrm{~mm}$ behind bregma, ML $3 \mathrm{~mm}$ from the midline suture, and between DV 12 and $16 \mathrm{~mm}$ below the level of the skull. Most of the lesions were made by passing $2 \mathrm{~mA}$ of current for $15 \mathrm{sec}$.

Following $24 \mathrm{~h}$ of recovery from the lesion, the rabbits were tested for retention of the short-delay CR with two blocks of paired training and one block of nine tone-alone trials. If the rabbit continued to show good CRs and UCRs, then he was given another lesion using the procedure described above. This procedure was repeated between one and seven times. If the rabbit showed no CR and a normal UCR, then he was put back into his cage and tested for retention on the following 2 days $(N=7)$. Several additional lesion animals $(\mathrm{N}=7)$ that showed substantial impairment of both the CR and UCR were not included in this study-it is difficult to interpret possible effects on the CR when the reflex pathways may have been damaged as well. There were five control lesions (see Results).

At the end of these procedures, the rabbits were overdosed with sodium pentobarbital and perfused intracardially with $.9 \%$ saline followed with $10 \%$ formaldehyde. The brains were postfixed for several days and then embedded in an albumin-gelatin mixture for later frozen histology. The brains were sectioned at $40 \mu \mathrm{m}$, mounted onto chrome-alum subbed slides, and dried, and every fourth and fifth section were stained for, respectively, cells (neutral red for Nissl substance and counterstained with potassium ferrocyanide for metal deposits) and fibers (Weil-Weigert). The extents of the lesions were determined by microscopic examination, and representative sections were drawn.

\section{RESULTS}

\section{Electrophysiological Recording}

Initial evidence that the pontine reticular formation may be involved in the learning circuitry for the conditioned response came from unit recordings. An example is shown in Figure 1A. The figure shows the averaged behavioral NM response from a block of eight paired trials in a well-trained animal, as well as the corresponding multiple-unit activity recorded from the more lateral pontine reticular formation approximately midway between the lateral lemniscus and the decussation of the brachium of the superior cerebellar peduncle. As can be seen, there is an evoked onset response to the tone CS and a suggestion of an evoked onset response to the corneal airpuff (either an auditory or tactile response). The unit firing may also follow the actual amplitude-time course of the NM behavior. Figure 1B is from another electrode located within the medial pontine reticular formation near the lateral edge of the decussation of the superior cerebellar peduncle in another animal. In this record, there is an increase in unit activity that clearly parallels the amplitude-time course of the learned and reflex components of the behavioral NM re- 

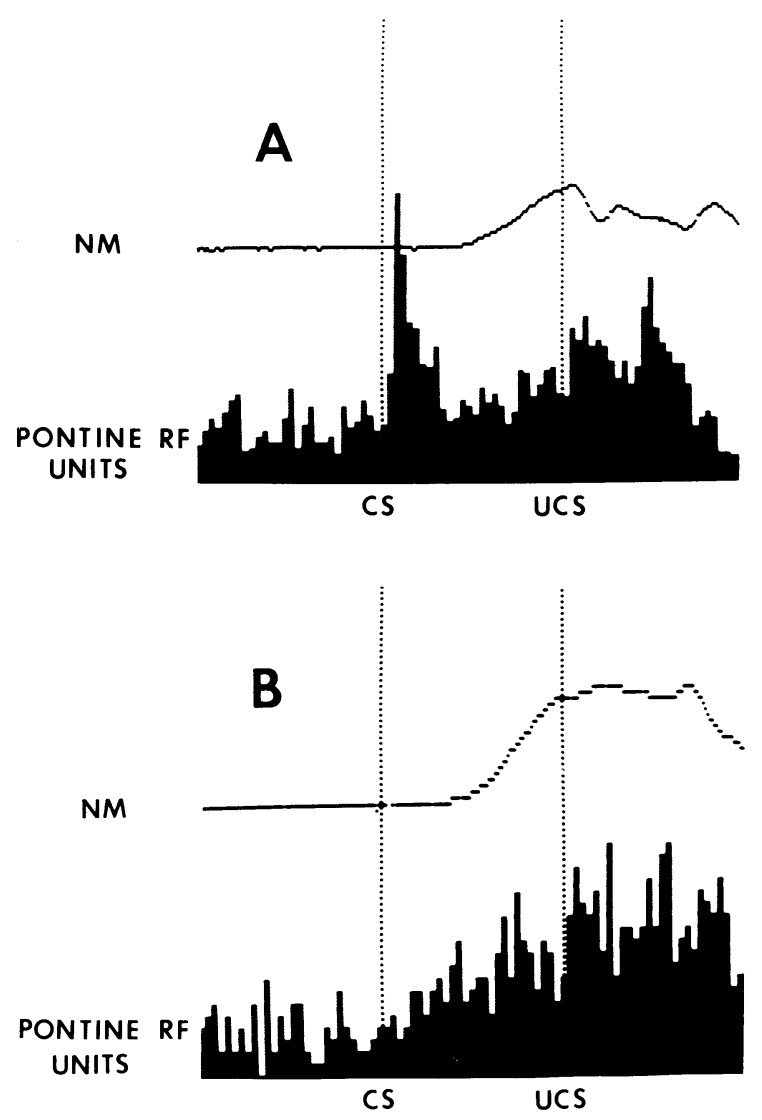

Figure 1. Computer-generated histogram of multiple-unit activity in the pontine reticular formation and the corresponding nictitating membrane (NM) behavior, both averaged over eight paired trials. The abscissa is 750 msec. Tone onset (CS) and airpuff onset (UCS) are marked by vertical lines. (A) Record from an electrode located midway between the lateral lemniscus and decussations of the superior cerebellar peduncle. (B) Record from an electrode within the lateral margin of the superior cerebellar peduncle.

sponse. This last component of the reticular unit response is similar to the learning-induced response that develops in the hippocampus (Berger \& Thompson, 1978) and to the responses found in the lateral portions of the ipsilateral cerebellum (McCormick et al., 1981). Thus, the pontine reticular formation shows a convergence of information concerning the presentation of the CS and the UCS, as well as a model of the learned behavioral response.

\section{Lesions}

The effect of the experimental lesion in the ipsilateral pontine reticular formation on the amplitudes of the conditioned and unconditioned responses is shown in Figure 2. The conditioned response is abolished in all seven animals. In marked contrast, the lesion has little effect on the unconditioned response. Separate repeated measures analyses of variance were completed for the CR and UCR. The lesion effect on the CR is highly significant $[F(3,18)=37.8, p<$ $.001]$. The mean amplitude of the $C R$ is virtually 0 for all 3 days of postlesion training-testing and is, of course, significantly lower than the prelesion amplitude. Five of the seven experimental lesion animals showed no clear evidence of conditioned responding postlesion, and two showed a slight degree of recovery-these two animals account for the fact that the postlesion CR amplitude is slightly above 0 . In marked contrast, although there is a small decrease in the UCR immediately after lesion of borderline significance in the seven animals $[F(3,18)=3.19, p=.5]$, that difference disappears completely by the third retention test [Scheffé test, $F(3,18)=.0018$, n.s.].

Figure 3 shows the composite histological reconstructions of the experimental animals at 9, 10, and $11 \mathrm{~mm}$ behind bregma. Note that the lesions typically have destroyed the region of the rostral pontine reticular formation just lateral to the decussations of the superior cerebellar peduncle. In fact, these lesions probably have destroyed the lateral margin of the decussation, as seen in the fiber stain, and therefore have interrupted efferents from the cerebellum. The lesion is in the region in which we have recorded stimulus-evoked responses and an increase in unit activity that is similar to the topography of the learned response (Figures $1 \mathrm{~A}$ and $1 \mathrm{~B})$. Figure 4 is a drawing of the rabbit within this group in which there was the smallest amount of damage. This animal showed no signs of a learned response in 3 days of retention tests. The lesion includes the medial pontine reticular formation that Brodal (1958) described as "motor."

Figure 5 is the composite drawing of the rabbits in which there was no attenuation of either the CR or the UCR $(\mathrm{N}=5)$. These lesions are generally more lateral to those of the former group and usually include portions of the lateral lemniscus and lateral

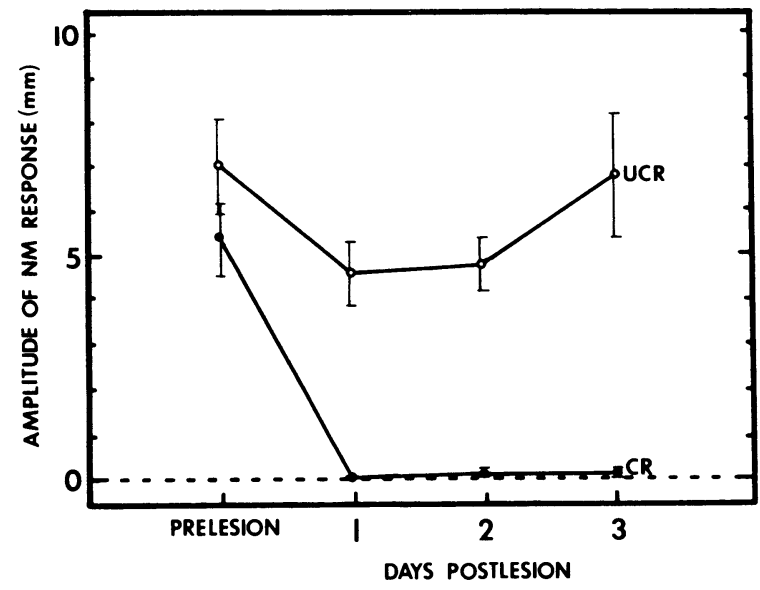

Figure 2. A comparison of the pre- and postlesion amplitudes (mean \pm standard error) of the NM responses in animals with pontine reticular lesions. After 3 days of retention tests, the reflexive UCR is at prelesion amplitude but the learned CR is lost. 


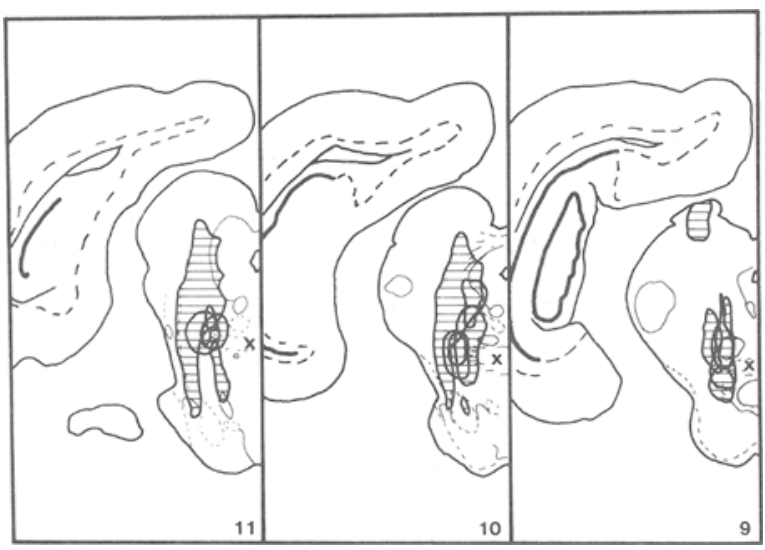

Figure 3. Composite drawings at 9,10 , and $11 \mathrm{~mm}$ behind bregma of the lesions that permanently reduced the learned $\mathbf{C R}$. The $\mathbf{X}$ denotes the decussations of the superior cerebellar peduncle. The reflexive response attained prelesion amplitudes.

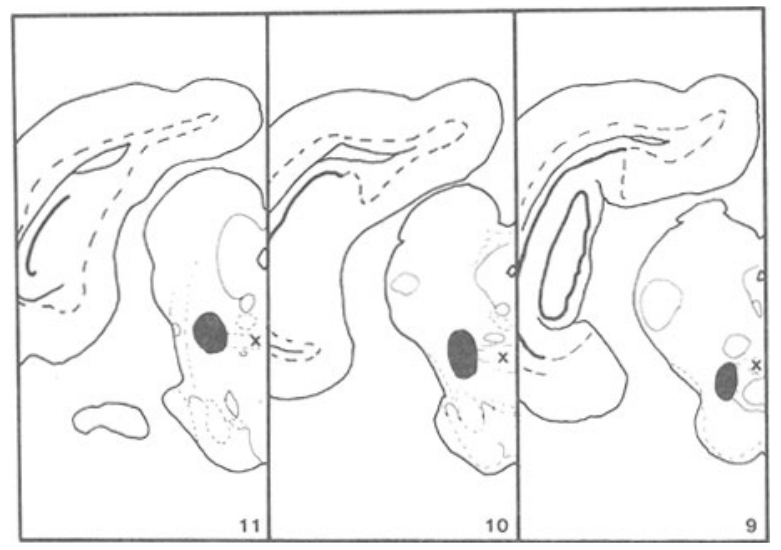

Figure 4. Drawings of the smallest lesion that effectively eliminated the learned $C R$. The $X$ denotes the decussations of the superior cerebellar peduncle.

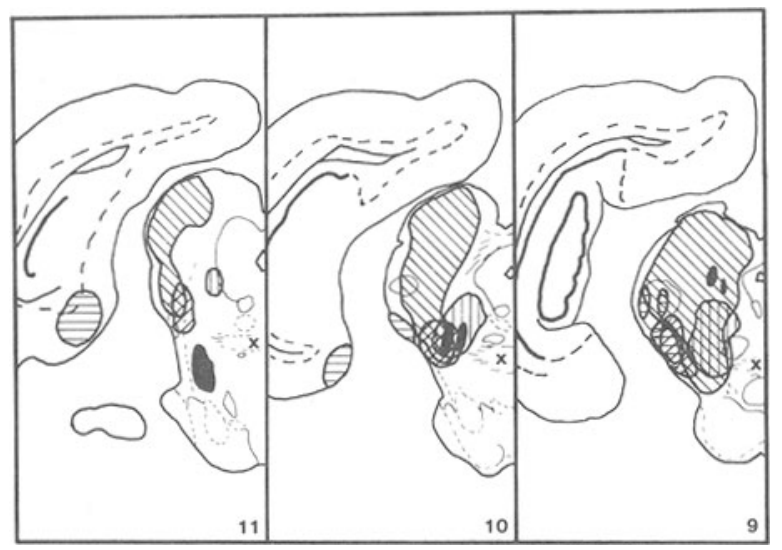

Figure 5. Composite drawings of the lesions in those rabbits in which there was no decrement in either the learned CR or the reflexive UCR. The $X$ denotes the decussations of the superior cerebellar peduncle. reticular formation and extend more dorsally as well, but do not include the region immediately lateral to the decussations of the superior cerebellar peduncle. These animals had no significant postlesion effect on either the $\operatorname{CR}[F(3,12)=1.8$, n.s.] or the UCR $[F(3,12)$ $=.8$, n.s.] when tested for repeated measures on the response amplitude. This control group tended to have more lesions (mean $=4.4)$ than rabbits within the experimental group (mean $=3.6$ ), although this was not a statistically significant difference $[\mathrm{t}(10)=1.5$, n.s.]. More important, the control lesion results indicate that brief anesthesia with halothane and electrolytic lesions per se do not account for the differences between the two groups.

\section{DISCUSSION}

The pattern of electrophysiological recording that we have found in the pontine reticular formation is what might be expected in a brain region that contains the essential neuronal plasticity coding a learned response: converging sensory information about the occurrence of the stimuli and a neuronal representation of the learned behavioral response. This latter component closely resembles the learning-induced response seen in the hippocampus (Berger \& Thompson, 1978); however, the hippocampus does not show stimulus-evoked responses. Because our initial recordings have been of multiple units, we do not yet know whether the various responses within each recording are generated by the same or different neurons. By the same token, we cannot be certain whether the unit discharges are from neuron somata or axons. These questions will be determined with future singleunit recordings. To date, we have seen the pattern of neural response described here for the pontine reticular formation in only one other structure: the cerebellum (McCormick et al., 1981). Damage to the ipsilateral cerebellum results in an abolition of shortdelay conditioned responding that is impervious to retraining (McCormick et al., 1981).

The experimental lesion rabbits of this current study as a group showed an essentially total retention deficit in conditioned responding, in marked contrast with the rabbits in the control group, who had no such deficits. Furthermore, the experimental lesion effect is selective in that it abolishes retention of the CR but has essentially no effect on the UCR. This in itself is an extremely important result. Thus, the circuitry essential for the learned response can be separated from the circuitry involved in the unconditioned reflex response. The most direct reflex pathwaystrigeminal afferents, interneurons, and motoneurons of at least the fifth, sixth, accessory sixth, and seventh cranial nuclei-therefore do not appear to be involved in the essential circuitry for the learned response.

At present, perhaps the most parsimonious explanation for our results is that reticular lesions have in- 
terrupted an essential cerebellar efferent. However, we do not know the nature of this efferent. It may be that the critical plasticity occurs in the cerebellum itself and the lesion has interrupted an essential output pathway for a cerebellar engram. Alternatively, the cerebellar pathway may provide a necessary input for the formation or expression of a reticular engram. Finally, neurons in both the pontine reticular formation and the cerebellum may be involved simultaneously in the neuronal plasticity that encodes the learned response. These possibilities will be determined in subsequent experiments. It should be noted that Moore (Note 5) found that lesions in a different region of the pons also abolish the rabbit NM conditioned response. The region of his effective lesion is also very close to the superior cerebellar peduncle.

One final point deserves emphasis. The present experiment demonstrated abolition of retention of the CR by ipsilateral reticular lesions. We do not yet know whether relearning can occur with extensive retraining. ${ }^{6}$ In the case of the cerebellar lesion, animals are permanently unable to relearn the CR with the eye ipsilateral to the cerebellar lesion. However, they relearn the conditioned NM and eyelid responses rapidly and robustly with the eye contralateral to the lesion (McCormick et al., 1981). These possibilities are now being examined for the pontine reticular lesion effect.

\section{REFERENCE NOTES}

1. Enser, D. Personal communication, July 26, 1976.

2. McCormick, D. A., Lavond, D. G., \& Thompson, R. F. A neurophysiological atlas of the brainstem components of classical conditioning of the rabbit's nictitating membrane response. Manuscript in preparation.

3. Lavond, D. G., Judson, S. M., \& Thompson, R. F. Simultaneous recordings from neurons of the accessory sixth and seventh cranial nuclei during classical conditioning of the nictitating membrane and eyelid responses. Manuscript in preparation.

4. McCormick, D. A., \& Thompson, R. F. Pattern of response of the facial musculature during classical conditioning of the nictitating membrane and eyelid response. Manuscript in preparation.

5. Moore, J. W. Personal communication, February 3, 1981.

6. Note added in proof. One additional animal was run with the lesion paradigm, and it was found that he could not relearn with 5 full days of retraining. The contralateral CR was learned and overtrained subsequently in 4 days. He was given an additional 5 days of retraining on the ipsilateral side (a total of 10 days of ipsilateral retraining) without showing learned behavior. The lesion for this animal was at the same location as those for rabbits with experimental lesions reported here.

\section{REFERENCES}

BACH-Y-Rita, P. Neurophysiology of eye movements. In P. Bach-y-Rita \& C. Collins (Eds.), Symposium on the control of eye movements. New York: Academic Press, 1971.

Berger, T. W., \& Thompson, R. F. Neuronal plasticity in the limbic system during classical conditioning of the rabbit nictitating membrane response. I. The hippocampus. Brain Research, 1978, 145, 323-346.

Brodal, A. The reticular formation of the brain stem. London: Oliver \& Boyd, 1958.

Cegavske, C. F., Patterson, M. M., \& Thompson, R. F. Neuronal unit activity in the abducens nucleus during classical conditioning of the nictitating membrane response in the rabbit, Oryctolagus cuniculus. Journal of Comparative and Physiological Psychology, 1979, 93, 595-609.

Cegavske, C. F., Thompson, R. F., Patterson, M. M., \& Gormezano, I. Mechanism of efferent neuronal control of the reflex nictitating membrane response in rabbit. Journal of Comparative and Physiological Psychology, 1976, 90, 411-423.

Gormezano, I., Schneiderman, N., Deaux, E., \& Fuentes, I. Nictitating membrane: Classical conditioning and extinction in the albino rabbit. Science, 1962, 138, 33-34.

McCormick, D. A., Lavond, D. G., Clark, G. A., Kettner, R. E., Rising, C. E., \& Thompson, R. F. The engram found? Role of the cerebellum in classical conditioning of nictitating membrane and eyelid responses. Bulletin of the Psychonomic Society, 1981, 18, 103-105.

Megirian, D., \& Bureš, J. Unilateral cortical spreading depression and conditioned eyeblink responses in the rabbit. $E x$ perimental Neurology, 1970, 27, 34-45.

Norman, R. J., Buchwald, J. S., \& Villablanca, J. R. Classical conditioning with auditory discrimination of the eye blink in decerebrate cats. Science, 1977, 196, 551-553.

OAKLEY, D. A., \& Russell, I. S. Neocortical lesions and classical conditioning. Physiology \& Behavior, 1972, 8, 915-926.

Papsdorf, J. D., Longman, D., \& Gormezano, I. Spreading depression: Effects of applying $\mathrm{KCl}$ to the dura of the rabbit on the conditioned nictitating membrane response. Psychonomic Science, 1965, 2, 125-126.

Salafia, W. R., Romano, A. G., Tynan, T., \& Host, K. C. Disruption of rabbit (Oryctolagus cuniculus) nictitating membrane conditioning by posttrial electrical stimulation of hippocampus. Physiology \& Behavior, 1977, 18, 207-212.

Solomon, P. R. A time and a place for everything? Temporal processing views of hippocampal function with special reference to attention. Physiological Psychology, 1980, 8, 254-261.

Solomon, P. R., \& Moore, J. W. Latent inhibition and stimulus generalization of the classically conditioned nictitating membrane response in rabbits (Oryctolagus cuniculus) following dorsal hippocampal ablations. Journal of Comparative and Physiological Psychology, 1975, 89, 1192-1203.

Thompson, R. F., Berger, T. W., Berry, S. D., Hoehler, F. K., Kettne R, R. E., \& Weisz, D. J. Hippocampal substrate of classical conditioning. Physiological Psychology, 1980, 8, 262-279.

Weisz, D. J., Solomon, P. R., \& Thompson, R. F. The hippocampus appears necessary for trace conditioning. Bulletin of the Psychonomic Society Abstract, 1980, 193, 244.

(Manuscript received June 30, 1981;

revision accepted for publication September 22, 1981.) 